\title{
Factors Contributing to the Late Commencement of Antenatal Care at a Rural District Hospital in Lesotho
}

\author{
Thandiwe Marethabile Letsie ${ }^{1} \&$ Matjeko Lenka $^{2}$ \\ ${ }^{1}$ Bloemfontein Free State South Africa \\ ${ }^{2}$ Matjeko Lenka, Elizabeth Glazer Paediatric Foundation Maseru, Lesotho \\ Correspondence: Thandiwe Marethabile Letsie, Bloemfontein Free State South Africa.
}

\author{
Received: January 27, 2021 Accepted: February 26, 2021 Online Published: March 30, 2021 \\ doi:10.5539/gjhs.v13n5p32 URL: https://doi.org/10.5539/gjhs.v13n5p32
}

\begin{abstract}
Antenatal care (ANC) is a key approach aimed at improving maternal and infant health. Numerous factors are associated with late commencement of antenatal care. Sub-Saharan Africa countries are exception to the problem of late commencement of antenatal care. The qualitative, explorative, descriptive and contextual approach was followed. The pregnant women meeting the inclusion criteria, were above 16 weeks and attended antenatal care at the time of the study. Different authorities granted permission to conduct face-to-face, unstructured in-depth interviews. Tesch approach enabled the qualitative researchers to immerse themselves through systematic organization and synthesis of data to create manageable units. An independent co-coder also analyzed data independently. Afterwards, they met and agreed on specific themes and sub-categories. The following five themes emerged; personal and family factors, cultural beliefs and practices, health systems and poor infrastructure. Measures aimed at improving accessibility to the health centers include; road infrastructure, telecommunication and more client centered services. Improvement of early commencement of antenatal services becomes an ideal approach influencing excellent maternal and neonatal outcomes. Therefore, government initiatives aimed at empowering communities on the benefits of commencing antenatal care on time is necessary.
\end{abstract}

Keywords: delay, commencement and antenatal care

\section{Introduction and Background}

Antenatal care remains the crucial entry point offering pregnant women a broad range of maternal health care promotion and preventative services. It is the umbrella term describing the relevant family centered health care services offered during pregnancy through screening, education and referral of complicated cases. The initiative has significantly reduced maternal and fetal mortality and morbidity rate globally (Cumber, Diale, Stanly, \& Monju, 2016; Oyerinde, 2013). About, 303,000 women and adolescents died in 2015 due to pregnancy, birthing related complications. Ninety-nine percent (99\%) of women predominantly from low resourced countries died, and approximately 2.6 million babies were stillborn in 2015. Between 2007-2014, only $64 \%$ of pregnant women attended minimal antenatal care of four contact sessions (The WHO report on recommendations on antenatal care for a positive experience, 2016). In Lesotho, the high maternal and infant mortality rate associated with pregnancy related complications still remain a concern. The maternal and child health program in Lesotho focuses on the following key issues: delivery, ante/postnatal care and childhood vaccination and treatment of common childhood diseases (Maternal and Child Health in Lesotho, 2019). This paper is keen in establishing factors contributing to the delay in commencing antenatal care in the mountainous parts of Lesotho.

The low and middle income economies have seen notable improvement in the attendance of antenatal care services since WHO introduced ANC model in 2002. The model offers a goal oriented approach aimed at delivering evidenced-based interventions carried out during each critical antenatal visitation period (The WHO report on recommendations on antenatal care for a positive experience, 2016). A more holistic health promotion and preventative approach driven through the Prevention of mother to Child HIV/AIDS initiative aims at improving maternal and neonatal health care outcome.

The difficult topography in the mountainous parts of Lesotho compromises desirable delivery of health care services at any given time. The mountainous country is divided into the north-western lowlands and south-eastern highlands with the altitude of 1800 metres above sea level. Sixty percent $(60 \%)$ of the country is mountainous and 
rural with minimal infrastructure compared to the lowlands. Access to basic health care services including antenatal care to rural communities is quite difficult, due to challenges like; transportation, poor telecommunication network and harsh weather (St James Mission hospital newsletter, 2021).

Lesotho has ten administrative districts located in different regions of the country, each having at least one hospital operating at different levels of care. St James mission hospital forming the context of this study is situated in Mant'sonyane within Thaba-tseka district. The district is located in the heart of Maloti Mountains about 125 kilometres away from the capital, Maseru. Four satellite health centres and posts forming the crucial referral points, surround the hospital. The nearby Mohale dam that is part of the joint Lesotho/South African highlands water project, has significantly improved the connection of the highlands with the lowlands through improved roads infrastructure and telecommunication. This has significantly improved the remote lifestyle experienced by rural communities in this district (St James Mission hospital newsletter, 2021).

The clinics and health care centres in Lesotho operating at the periphery, form crucial primary entry point into district hospitals in both urban and rural areas. The third level of care offered at tertiary care, treats complicated conditions within different specialities like; paediatrics, obstetrics and gynaecology, internal medicine and surgery. More complicated conditions requiring more specialized care are referred to more advanced academic hospitals in the Free State (Lesotho Ministry of Health, Health PHC revitalization plan 2011-2017).

The maternal mortality ratio in Lesotho estimated at 1,155 deaths per 100,000 live births between1990-2015 puts the country among the highest in the region. Proactive measures aimed at reducing maternal deaths to 300 deaths per 100,000 live births by 2015 have been implemented by Lesotho government (Satti, Motsamai, Chetane, Leshoboro-Marumo, Barry, Riley, McLaughlin, Seung, \& Mukhere, 2012). One of the key approach aimed at reducing maternal mortality and morbidity is attainable through provision of cost-effective antenatal care, thereof making it more accessible to women (Price Water Coopers, Health system Innovation in Lesotho, 2013).

Commencement of antenatal care in Lesotho differs in different regions, namely the highlands and lowlands. For instance, the mountainous areas of the country usually experience harsher living conditions worsened by extremely cold weather due to the rugged mountainous terrain. Accessibility of health care facilities in the heart of maloti mountains is difficult except through horse backs (St James Mission hospital newsletter, 2021).

Antenatal care attendance in Lesotho like in many other countries is strongly associated with high levels of education (Gill, Hoffman, Tiam, Mohai, Mokone, Isavwa, Mohale, Matela, Ankrah, Luo, \& Guay, 2015). People living in the lowlands in Lesotho, are generally better educated than their counterparts living in the highlands. Women with better education, are more likely to receive the four antenatal sessions recommended by WHO in comparison to the less educated ones. Availability of resources is in more urban settings offers women better opportunities and they become more financially independent. This is a positive influence, enabling women to commence antenatal care on time (The Lesotho Ministry of Health demographic and health survey report, 2014).

In the more rural high lands regions of Lesotho, men have less opportunities of education compared to those residing in urban, low-land districts. For instance, only $21 \%$ of men living in highlands districts are literate compared to $50 \%$ of their counterparts residing in lowlands districts. As a result, women residing in the highlands areas of Lesotho tend not to be adequately supported by their partners who are ignorant on different reproductive related issues (The Lesotho Ministry of Health Demographic and Health Survey report, 2014).

The stigma associated with teenage pregnancy is another obstacle associated with late commencement of antenatal attendance. This becomes a major concern especially among adolescents and younger women. Separate private facilities become a meaningful option enabling pregnant adolescents to access the services without experiencing immense stigma (Pell, Men, Were, Nana, Afrah, Chatio, \& Poo, 2013). Adolescent Health Corners offering different services tailor-made to specific needs of the young women address their diverse needs. Such facilities are gradually becoming popular in many countries including Lesotho. The reproductive health care corners for younger mother are intended to offer a range of sexual and reproductive health care needs including; antenatal care services offered to the adolescents through education, and counseling programs. The unique facilities offer needed privacy to younger women, who usually do not feel comfortable to receive joint services with older clientele (Gill et al., 2015; Matobo, Makatsa, \& Obioha, 2009).

Certain cultural myths, including witchcraft within the Basotho culture are quite influential in delaying commencement of antenatal care on time. The rural, less educated women tend to conceal the early stages of pregnancy due to fear that they might be bewitched pregnancy (Semenya \& Letsosa, 2013; Ochako et al., 2011).

The extended family setup becomes quite influential in determining desirable antenatal attendance within the Basotho culture. The young married couple usually resides at the groom's patriarchal home under the parents' 
authority. This arrangement becomes even more evident, where a young man is sometimes compelled to seek employment in the lowlands of Lesotho or even to the neighboring South Africa (Matobo, et al., 2009; Gill et al., 2015). The remaining mother, has to plan her needs around the routine of her in-laws. The extensive routine including normal home chores of cleaning, washing and cooking for the extended family and even farming related duties can be quite daunting for the pregnant young woman making it difficult to find time to attend antenatal care. The complicated extended family setting, compels the pregnant woman to seek consent from the husband or in-laws to commence antenatal care (Gill et al., 2015).

Poor antenatal care attendance if further compounded by attitudes of service providers. The unprofessional behavior of health care providers tends to impact negatively on health service utilization. The unappealing behavior of some midwives who are reputable to being harsh, unfriendly, rude and careless during antenatal, labor and post-natal periods causes gross dissatisfaction to some pregnant women (Chiwaula, 2011).

The dawn of HIV/AIDS has significantly influence attendance of antenatal care especially in the Sub-Saharan countries due to the associated stigma of the disease (Turan, Hatcher, Medema-Wijnveen, Onono, Miller, Bukusi, Turan, \& Cohen, 2012). The HIV/AIDS infection in pregnancy poses a greater risk to the unborn baby likely to get infection through vertical transmission. In Lesotho, (27.7\%) of HIV/AIDS prevalence rate is concerning. The high prevalence rate of HIV/AIDS infection affecting everyone, associated with immense stigma amongst concerned couples/partners acts as a deterrent for antenatal care attendance. (Lesotho Ministry of Health, PMTCT guidelines, 2017).

Excessive waiting time in the antenatal health care becomes a major barrier affecting proper adherence to antenatal care attendance (Ekabua \& Njoku, 2011). The current disease burden from both communicable and non-communicable illnesses is putting so much pressure on PMCTC program nurses who are usually quite few in numbers. As a result, prolonged waiting time in antenatal care clinics has become a norm especially in most public health care facilities offering services on the ground. The lack of resources especially labor becomes a serious setback to employed pregnant women who are obliged to receive services from the disgruntled and insensitive nurses experiencing numerous challenges including poor staffing. Consideration of creative solutions accommodating working people, in quite sensible (Sibiya, Ngxongo, \& Bengu, 2018).

\section{Methods}

\subsection{Study Design}

The subjective and systematic nature of qualitative studies bring in life experiences through description of the unique encounters enabling the researcher to explore the depth, richness and complexity of a phenomenon being discussed (Burns \& Grove, 2009; Creswell, 2009). The qualitative study was explorative, descriptive and contextual in nature. This shed light on the thoughts and behaviours of participants concerning late commencement of antenatal care (Boyce \& Neale, 2006).

\subsection{Study Setting}

St James mission hospital has a 60 bed capacity. A number of health care centers and clinics offering Primary Health Care services refer complicated cases requiring further management. The fully operational departments within the hospital, offer the following services; out patients, dental and pharmacology. Tertiary and allied social development services are also offered (St James mission hospital. The Anglican Diocese of Lesotho, 2021). In-depth face-to-face unstructured interviews were conducted at a private venue of the antenatal care clinic.

\subsection{Population and Sampling}

The population of the study constituted by all pregnant women, meeting the inclusion criteria, above 16 weeks of gestation and commenced antenatal care within the specific month of the study (Polit \& Beck, 2012). The non-probability purposive sampling technique used in the selection of the sample, revealed those significant factors contributing to the late commencement antenatal care.

\subsection{Data Collection}

In-depth face-to-face unstructured interviews were conducted with participants coming from the surrounding rural villages within St James Hospital catchment area. The necessary arrangements with the authorities of the hospital were made prior entry into antenatal care clinic setting. A suitable venue offering privacy improved interaction and the quality of interview sessions. Prior conduction of each in-depth face-to-face unstructured interview session, the purpose and the other technicalities of the study were introduced, during briefing exercise. Voluntary participation in the study was emphasised and those agreeing to participate, signed the consent form. This question formed the basis of interviews: "What are the contributing factors towards your delay in commencing antenatal care at this 
clinic?" The following qualitative interviewing techniques considered by the researcher included; probing, reflecting clarifying and paraphrasing (Polit \& Beck, 2012). The interactive approach enabled the participants to engage more freely with the researcher through integrating phrases like; please clarify this issue more, did you mean this, Oh! This make sense (Polit \& Beck, 2012). The participants were cautioned that in-depth face-to-face unstructured interviews would be tape-recorded. The researcher's good communication skills facilitated the desirable flow of the discussions through striking a good balance between talking and listening (Ngomane \& Mulaudzi, 2010). The researcher took cognizance of relevant non-verbal cues that could reflect discomfort from the participants and so on (Ngomane \& Mulaudzi, 2010). The in-depth nature of interviews enabled the participants to discuss their concerns and challenges encountered with ease. The interviews were conducted in Sesotho which is the language commonly spoken and the principle of data saturation was considered. After each face-to-face unstructured in-depth interview session, a debriefing exercise was initiated to get further gain clarity on any outstanding issue.

\subsection{Data Analysis}

The analysis of qualitative data does not necessarily follow a specific trend. Cautious synthesis, multi-layered in nature, builds upon itself till a meaningful, contextual-derived, and verifiable interpretation is attained (Roller \& Lavraks, 2015). The similarities, inconsistencies and close-knit contextual input obtained in qualitative studies affirm the inductive stance that builds up from inside out, through analytical interpretations culminating in meaningful themes (Roller \& Lavraks, 2015). Analysis of data in this study followed Tesch's approach. Vast data elicited was transcribed from Sesotho into English. It was read thoroughly; similar topics were identified, those matching were abbreviated into codes; most descriptive wording of topics was checked and turned into major themes/categories; related topic grouped together to diminish total list of categories, final decision on codes and written in a sequential order (Creswell, 2009).

\subsection{Trustworthiness}

Trustworthiness seeks to establish confidence on the data collected. Qualitative researchers adopt this technique through adhering to the four principles of truth and value by Lincoln \& Guba; credibility, confirmability, dependability, and transferability (Creswell, 2009; Polit \& Beck, 2012).

\subsection{Ethical Consideration}

Prior commencement of the study permission the following authorities gave ethical clearance: Health Science Faculty, University of the Free State, Ethics Committee Department of Health Lesotho, administration of the hospital and antenatal clinic and the participants. The following ethical principles were held; beneficence, confidentiality, informed consent and justice.

\section{Results}

The elaborate transcription of data culminated in the following five themes each with its sub-category: personal factors, family, cultural beliefs and practices, health systems and poor infrastructure.

Table 1. Outcomes of late commencement of antenatal care

\begin{tabular}{|c|c|c|}
\hline Themes & & Sub-categories \\
\hline 1. & Personal factors & 1.1 literacy level \\
\hline 2. & Family factors & 2.1 Attitudes of in-laws \\
\hline \multirow{3}{*}{3.} & \multirow{3}{*}{ Cultural beliefs and practices } & 3.1 Age \\
\hline & & 3.2 Parity \\
\hline & & 3.3 Marital status \\
\hline \multirow{5}{*}{\multicolumn{2}{|c|}{ 4. Health systems }} & 4.1 Compulsory HIV testing \\
\hline & & 4.2 Compulsory accompaniment by husband \\
\hline & & 4.3 Cost of antenatal care \\
\hline & & 4.4 Slow service \\
\hline & & 4.5 Service provision by male nurses \\
\hline 5. & Poor infrastructure & 5.1 Inaccessible health care facilities \\
\hline
\end{tabular}




\subsection{Personal Factors}

The low literacy rate is one the personal factors influencing commencement of antenatal care in the highland regions of Lesotho.

\subsubsection{Literacy Level}

Literature affirms that people living in the lowlands regions are generally better educated than counterparts living in the highlands regions which are more rural. For instance, the proportion of women with at least some secondary education ranges from $29 \%$ in Thaba-tseka (highlands) to as high as $61 \%$ in one of the lowlands districts.

The participants affirmed the issue of poor literacy level of people residing in the mountainous regions of Lesotho:

“.....We are mountainous people, so we are not well informed on a number of issues. As a result, we lack adequate knowledge and consequences thereof of not commencing antenatal care on time”.... (Participant \# 1).

\subsection{Family Factors}

The extended family setting, commonly found within most African cultures offers experienced family members to share their reproductive related experiences that assists the pregnant mother during pregnancy, childbirth and antenatal period (Haobijam, Sharma, \& David, 2010).

\subsubsection{Attitudes of In-Laws}

Decision to attend antenatal care in certain culture depends on the support by the family especially mother-in law who takes charge of domestic responsibilities. The young mother has to assists the family with common chores, before being permitted by the mother in-law to attend antenatal care (Simkhanda, Porter, \& Van Teijlingen, 2010).

The participants confirm the existing lack of decisive power by pregnant Basotho women concerning their reproductive lives:

"Sometimes mothers-in-law is not pleased when one plans to go to the clinic due to daily numerous domestic Chores". (Participant \# 1).

\subsection{Cultural Beliefs and Practices}

In Lesotho, similar culturally related fears regarding witchcraft tend to affect the announcement of the pregnancy (Semenya \& Letsosa, 2013). The statements below confirm the participants' fear of commencing antenatal care services on time due to cultural stereotypes associated with witchcraft:

"One is not keen to commence antenatal due to fear that one will be bewitched". (Participant \# 6).

One participant recognizes the significant role played by traditional healers in treating rural communities through tapping in their enormous knowledge of indigenous medicine:

"We belief that even if one does not attend antenatal care, the traditional medications prescribed by our traditional healers still help us a lot." (Participant \# 1).

\subsubsection{Age}

Most primary health care clinics do not separate antenatal care services according to age due to lack of resources (Matobo et al., 2009). The following statements confirm the participants' concerns on maternal young age as being key:

"Sometimes being very young and pregnant, one will find that they are alone among adults." (Participant \# 5).

\subsubsection{Parity}

Women with several children tend to rely on their experiences from previous pregnancies. More experienced women with many children that there is no need to attend antenatal care or be ridiculed by nurses due to improper child spacing (James et al., 2012). Poor child spacing posing as an impediment was raised by one participants who said:

"Sometimes when a woman becomes pregnant frequently, she becomes shy to make the pregnancy known by going to the clinic due to fear of being ridiculed by nurses." (Participant \# 6).

\subsubsection{Marital Status}

In Lesotho, pregnancy out of marriage is quite shameful to the girl as well as her parents (Resty, 2011). One participant affirmed the shame;

"It becomes one embarrassing situation influencing the unmarried girl not to attend antenatal care on time. (Participant \# 7). 


\subsection{Health Systems}

Improving maternal and child health is a priority in many countries including Lesotho through making services accessible to all women despite their socio-economic background.

\subsubsection{Compulsory HIV Testing}

Routine HIV Voluntary Counselling and testing for pregnant women and their partners enables them to gain access to HIV treatment and other preventative interventions received through PMTCT initiative.

One major impediment echoed by different participants was fear of testing for HIV.

“...Both we as pregnant women and our partners fear to test for HIV. Such a test usually creates serious conflicts in our relationships, especially if one test positive and one negative." (Participant \# 1).

\subsubsection{Compulsory Accompaniment by Husband/Partner}

The husbands are usually unavailable to accompany their wives to the antenatal care clinic due to work commitments and even those who are available do not necessarily support idea because it is purely female responsibility.

The pregnant women raised their concerns regarding accompaniment by partners:

“...Sometimes you will find that the husband is not staying at home due to work commitments. The rule of the clinic is that we should come along with husbands to clinics." (Participant \# 2).

\subsubsection{Cost of Antenatal Care}

Lack of money due to unemployment is a key barrier to some women to access antenatal care on time. One participant raised the lack transport or minimal services money as a serious impediment:

"There is no money to pay for the user fees of R15.00 and transport. Some of us are unemployed" (Participant \# 2).

\subsubsection{Slow Service Delivery}

The slow services at some of these clinics create risk for the women who have to travel back home sometime alone in quite dangerous terrain. One participant echoed the concern of slow services:

"...In addition, long waiting for the services at the clinic is another factor. It means that one has find someone to accompany them when they finish late at the clinic..." (Participant \# 6).

\subsubsection{Service Provision by Male Nurses}

Within the Basotho culture, incorporating male nurses into such a female dominant profession still creates a lot of discomfort amongst Basotho women (Yanikkerem, Ozdemir, Bingol, Tatar, \& Karadeniz, 2009). One participants shared their discomfort around being examined by male nurses:

"One removes clothes in front of male nurses at the clinic, to be examined, they insert fingers. This practice is not acceptable for us as Basotho..." (Participant \# 7).

\subsection{Poor Infrastructure}

The poor roads, insufficient telecommunication network and impaired transportation becomes a major obstacle affecting meaningful antenatal care services (Onta, Choulagai, Shrestha, Subedi, \& Bhandari, 2014).

\subsubsection{Inaccessibility of the Health Facility}

Accessibility to health care for remote communities with minimal infrastructure still remains key challenge especially during the winter season. The concern of meaningful access to the antenatal care clinics was raised by a participant:

"Most of us are staying far away from the health services".

Clinics are very far and we are not able to start antenatal care on time..." (Participant \# 6).

\section{Discussion of Results}

Effective antenatal care management within the first trimester influences desirable outcomes (Ndidi \& Osereman, 2010). Different reports from developed countries like the United Kingdom, identify late commencement of antenatal as a significant risk factor for maternal deaths across different racial groups (Haddrill, Jones, Michell, \& Anuba, 2014). The indecisiveness of the mother in discontinuing or keeping the pregnancy at the on-set, ultimately resulting concealment of pregnancy, jeopardizes the desirable obstetrical and neonatal outcome (Siyange, Sitali, Jacobs, Musonda, \& Michelo, 2016). Extensive analysis of qualitative data culminated in five emerging themes; 
personal factors, family related factors, cultural and beliefs and practices, health systems and poor infrastructure.

A number of personal factors significant in contributing towards delayed antenatal care are highly individualistic, thereof unique. In the context of this study, low literacy level of pregnant mothers came out as critical influence. For instance, women with better education are more likely to attend the minimal recommended four sessions by WHO. In rural parts of Lesotho, even educated women still recognize the significant position of husbands and their notable leadership role in family related decisions (Gill et al., 2015). The education disparity between the high and low lands districts of Lesotho reveals the variance between Thaba- tseka district (highlands) in which $29 \%$ of women have obtained minimum secondary education contrary to $61 \%$ seen in the Lowland district of Berea (Gill et al., 2015). "We are mountainous people, so we are not well informed on a number of issues as a result we lack adequate knowledge."

The family centred approach is a preferred means of successful delivery of antenatal care services through initiatives such PMCTC. The extended family setup in rural Lesotho has a number of cultural stereotypes affecting timeous access to antenatal care. Elderly women as experienced traditional birth attendants, play a significant role in influencing decisions on issues of pregnancy, birthing and puerperium. Therefore, they tend to be sceptical to encourage younger pregnant women to commence antenatal care at designated health care facilities where services offered by a midwife are available (Edmonds, Paul, \& Sibley, 2011). "Sometimes the mother in-law tends to discourage us from listening to nurses' advice because they feel that they are equally experienced."

Basotho stereotypical lenses concerning the cultural practices around pregnancy, birthing and postnatal care practices, influence desirable antenatal follow-up to a certain extent (Breckenridge, Deveney, Kroll, Lazenbatt, \& Taylor, 2014). For instance, the myths of witchcraft remain a serious concern influencing the disclosure of pregnancy to health care workers who are not members of the family especially in the rural areas of Lesotho (Semenya \& Letsosa, 2013). This denies a pregnant mother opportunity of screening, treatment and referral if the need arise (Lesotho Government Strategic Plan, 2013). "One is not keen to commence antenatal due to fear that one will be bewitched".

The socio-demographic dynamics such as maternal age, parity and marital status also have an impact on a mother's decision to start antenatal care. The stigma associated with pregnancy out of the wedlock especially on teenage girls contributes to delayed commencement of antenatal care (Pell et al., 2013). The dawn of civil rights movement in different countries worldwide brings a remarkable milestone, where health care systems are embarking on measures ensuring utmost privacy of all the women including grand multiparous women scared of service providers' criticism on poor family panning practices seen through frequent pregnancies. "Sometimes when a woman becomes pregnant frequently, she becomes shy to make the pregnancy known by going to the clinic due to fear of being ridiculed by nurses."

Pregnancy out of the wed-lock common to young pregnant mothers exposes vulnerable women to societal criticism and isolation (Pell et al., 2013). In different countries, the notion of protection of young mothers is quite central to preventative reproductive health care programs (Matobo et al., 2009). Reproductive services offering needed privacy to the teenage mothers aligned to the mainstream reproductive health care basket are preferred. The protective and preventative approach where the teenagers get individual counselling tends to alleviate social consequences of repeated adolescent pregnancies culminating in the exclusion of young pregnant girls from the mainstream education system (Pell et al., 2013; Matobo et al., 2009). The pregnant young woman usually fears community especially adults and friends on what they will say about pregnancy outside marriage".

Access to meaningful antenatal care is the right to every mother. The strong antenatal care policy is an anchor to any progressive health care system. A client-centred approach where prompt services are offered attracting working clientele is meaningful. Cognizance on issues of gender related stereotypes relevant to the Basotho culture on reproductive related matters brand the services to a widest clientele. Family centred facilities inviting participation by partners are preferred. It is sensible to create polices accommodating even women who cannot afford the cost of such services and make such facilities accessible to all women across the spectrum. In different, rural parts of Africa including Lesotho, pregnant women still travel lengthy distances between three to four hours on foot to access the nearest health care facility. Clinics are very far and we are not able to start antenatal care on time..."

Sometimes tardiness in manner in which the services by despondent staff is not only a time waster, but pose as a danger to women who have to travel alone long distances on foot in areas which still do not have good transport (Sanda, 2014). Such risky terrains expose the pregnant women for attacks by wild animal, psychopathic personalities who sometimes rape and kill these vulnerable women (Andrew, Angwin, Auwun, Aniels Mueller, Phuanukoon, \& Pool, 2014). "In addition, long waiting for the services at the clinic is another factor". 
The lack of employment opportunities in the rural parts of Lesotho disadvantages women residing in the heart of maloti mountains with minimal resources to receive antenatal screening on time. Some women who do not have transport money cannot afford the minimal consultation fee of fifteen Maloti covering overheads for each antenatal visit. This prevents them access to antenatal care services on time. "There is no money to pay for the user fees of R15.00 and transport."

The dawn of HIV/AIDS has brought even more scepticism for many rural pregnant women in many parts of the world to commence antenatal care on time (Bernstein, Marcus, Nieri, Philip, \& Klausner, 2010). Despite the inception of Prevention of Mother to Child Transmission programs (PMCTC) recommending more family centred approach, there is still gross apathy among younger and older pregnant couples to attend antenatal care which recommends intense initial screening for communicable diseases like HIV/AIDS. The fear of testing HIV positive tends to bring irreconcilable conflicts among couples (Shamu, Zarowsky, Shefer, Temmerman, \& Abrahams, 2014). In many rural communities, where younger families do not consistently reside together due to work commitments by male partners, the separation creates temptation to both males and females to engage in extra marital sex. The subtle uncertainty on the couples' HIV status becomes a deterrent for commencement of antenatal care on time. "We fear to go for inclusive HIV testing at the antenatal clinic. It creates irreconcilable conflicts between couples."

A notable milestone by many governments saw health care services provided by male nurse practitioners in different African countries. This initiative has broken the long-standing gender stereotypes within a predominantly female nursing profession. However, the findings of study conducted by Yanikkerem et al., (2009) on women's attitudes and expectations regarding gynaecological examinations revealed more preference to female gynaecologists/midwives versus male health care practitioner, due to too much exposure during such examinations. This practice causes great discomfort to more conservative rural Basotho women. "One is removing clothes in front of male nurses at the clinic, for physical examination, they insert fingers."

Lack of good infrastructure in rural communities of Lesotho, makes health care services inaccessible (Onta et al., 2014). The poor road infrastructure, ineffective telecommunication and lack of transport in some villages, contributes to the delay in commencing antenatal care services. "Clinics are very far and we are not able to start antenatal care on time..."

This paper attempts to reflect on the ongoing plight of rural women who still experience immense challenges frustrating access to antenatal care. For instance, rural women still travelling long distances to clinics, lack resources, constant skeleton staff over-worked due to a more comprehensive delivery of Primary Health Care services approach operating with meagre resources, unwelcoming attitudes, long waiting times and lack of transport money to the clinic and socio-cultural practices influenced by beliefs like witchcraft (Sibiya et al., 2018).

A picture painted through these findings, being systems inclined is quite insightful. The collectiveness of different stakeholders like; families, communities and political affiliates externally, influences the health care systems to integrate progressive antenatal care services policies. It is sensible to improve road infrastructure, telecommunication and health care facilities to make services more accessible to rural communities of Lesotho. Internally, it is sensible to consider quality improvement systems addressing the following issues; a more transformative internal policy, taking heed of pertinent ethical issues like; confidentiality, privacy, justice and gender related issues.

The findings are quite informative, but limited. Other scholars bring in a broader perspective on the findings, through adding other significant influences into three broad categories namely; socio-demographic, socio-economic and biological factors. The socio-demographic factors emphasise the need for availability of resources in order to deliver quality improved antenatal care services. The supportive family structure allows women to commence antenatal care on time. Socio-economic factors being equally significant include; educational level of a woman, wealth level and their current employment status. Lastly, the biological factors equally being crucial determinants consider aspects such as; first pregnancy, current age, previous foetal loss and so on (Sinyange, Sitali, Jacobs, Musonda, \& Michelo, 2016).

\section{Conclusions}

Lesotho still experiences challenges of gross shortage of supplies especially in rural clinics, in certain instances. The delays in the ordered supplies including drugs becomes worse in the freezing winter months and it becomes difficult to transport medicinal supplies by road or air. The lack of supplies discourages women to commence antenatal care on time (Satti et al., 2012). Notable concerns affecting rural women in Lesotho are heighted by geographic and economic barriers drastically affecting access of women to antenatal care services (Satti et al., 
2012). In rural settings of Lesotho, the traditional birth attendants still play a significant role in antenatal care, delivery and puerperium. The indigenous attendants, forfeit a commission if they do not assist women to deliver personally. As a result, they tend to be quite skeptical to refer women for antenatal care in conventional clinics where women are screened and examined and referred if necessary. Such practice could be quite detrimental to the mother and child (Satti et al., 2012).

\section{Recommendations}

\subsection{Improve Resources}

Implementation of effective and comprehensive health care programs in rural settings depends on availability of resources. Improving resources such as road infrastructure and other health care resources in the difficult mountainous areas eases the referral process for critical emergencies for transfer to more advanced tertiary health care institutions in the low lands of the country.

\subsection{Collaborative Approach with Traditional Medicine}

A more positive approach sets a common ground for lasting partnerships between more scientific bio-medical approaches with existing Basotho socio-cultural practices influencing meaningful antenatal care commencement through a comprehensive family centred approach.

\subsection{Taking Cognizance of Gender Preferences in Different Region by Leadership}

The significant role played by male nurse is notable stride achieved by health care system in Lesotho and other countries globally through integrating the engendered nurses in different nursing specialities inclusive of obstetrical care. However, in rural settings, cultural stereotypes males and female nurses' roles call for health care leadership that that is quite sensitive towards cultural beliefs and practices of specific communities when allocating nurses.

\subsection{Community Partnership with Local Government}

Establishment of community partnerships with local government including chiefs to discuss any incidents that resulted in foetal and maternal deaths including those relating to professional mal-practice is quite sensible. Such positive platforms offer relevant parties' opportunities to agree on meaningful strategies aimed at improving the delivery of reproductive health care services.

\subsection{Health Education Campaigns}

On-going health education campaigns on prevention of teenage pregnancy and communicable diseases like; HIV/AIDS through mass media and other avenues is quite essential. It is important for the Ministry of Health in Lesotho to promote inclusive reproductive health services to rural families through accommodating working males (looking either after animals or migrant labourers or those working in the low lands).

\section{Limitations of the Study}

- The women who commenced antenatal care before 16 weeks were not included;

- Some participants were hurried to complete interviews because they had to travel back home alone before sun set.

- Women meeting the inclusion criteria were limited

- The biographical information was not elicited from the participants.

\section{Competing Interests Statement}

The authors declare that there are no competing or potential conflicts of interest.

\section{References}

Andrew, E. V. W., Pell, C., Angwin, A., Auwun, A., Daniels, J., Mueller, I., ... \& Pool, R. (2014). Factors Affecting Attendance at and Timing of Formal Antenatal Care: Results from a Qualitative Study in Madang, Papua New Guinea. PLoS ONE, 9(5), 1-14. https://doi.org/10.1371/journal.pone.0093025

Bernstein, K. T., Marcus, J. L., Nieri, G., Philip, S. S., \& Klausner, J. D. (2010). Rectal Gonorrhea and Chlamydia reinfection is associated with increased risk of HIV seroconversion. Journal of Acquired Immune Deficiency Syndrome, 53(4), 537-543. https://doi.org/10.1097/QAI.0b013e3181c3ef29

Boyce, C., \& Neale, P. (2006). Conducting in-depth interviews: A guide for designing and conducting In-depth Interviews for Evaluation Input. Retrieved from www.pathfinder.org/site/docserver/m-c-tool-series.PDF 
Burns, N., \& Grove, S. K. (2009). The practice of nursing research. Appraisal, synthesis, and generation of evidence (6th ed.). Saunders Elsevier. St Louis, United States of America.

Chiwaula, C. H. (2011). Factors Associated with Late Initiation of antenatal care (ANC) among women of Lilongwe. Retrieved from http://www.medcol.mw/commhealth/mph/dissertations/Catherine $\% 20$ Chiwaula\% 20corrected\%20revised\%20version.pdf

Creswell, J. W. (2009). Research Design: Qualitative, Quantitative, and Mixed Methods Approaches (3rd ed.). Sage Publications, Inc. Los Angeles.

Cumber, S. M., Diale, B. M., Stanly, E. M., \& Monj, N. (2016). Importance of Antenatal services to pregnant women at Buea Reginal hospital Cameroon. Journal of Family Medicine and Health Care, 2(4), 23-29. https://doi.org/10.11648/j.jfmhc.20160204.11

Ebonwu, J., Mumbauer, A., Uys, M., Wainberg, M. L., \& Medina-Marino, A. (2018). Determinants of late antenatal care presentation in rural and; peri-urban communities in South Africa: Cross-sectional study. Journal PLOS/ONE. https://doi.org/10.1371/journal.pone.0191903

Ekabua, J., Ekabua, K., \& Njoku, C. (2011). Proposed framework for making focused antenatal care services accessible: A review of the Nigerian Setting. https://doi.org/10.5402/2011/253964

Gill, M. M., Hoffman, H. J., Tiam, A., Mohai, F. M., Mokone, M., Isavwa, A., .. \& Guay. (2015). Pregnant and postpartum women's experiences and perspectives on the acceptability and feasibility of copacked medicine for antenatal care and PMCTC in Lesotho. https://doi.org/10.1155/2015/435868

Haobijam, J., Sharma, U., \& David, S. (2010). An exploratory study to assess the Family support and its effect on outcome of pregnancy in terms of maternal and Neonatal health in a selected hospital, Ludhiana Punjab. Nursing and Midwifery Research Journal, 6(4), 137-145. https://doi.org/10.33698/NRF0116

James, S., Rall, N., \& Strumpher, J. (2012). Perceptions of pregnant teenagers with regard to the antenatal care clinic environment. https://doi.org/10.4102/curationis.v35i1.43

Lesotho Ministry of Health. (2014). Demographic and Health Survey 2014. Retrieved from http://www.dhsprogram.com/pub/pdf/FR309.pdf.

Lesotho Ministry of Health. (2017). Lesotho PHC revitalization plan. Retrieved from http://www nationalplanningcycles.org/default/files/country/-docs/Lesotho/Lesotho-phc-action-2011-2017-draft-submitt ed-t0-moh-325

Lesotho Ministry of Health. (2013). National Guidelines for Prevention of Mother to Child Transmission of HIV (3rd edition). Maseru, Lesotho: Ministry of Health. Retrieved http://app.who.int/medicinesdoc/document/s/9148en/s19148en.pdf.

Lincoln, Y. S., \& Guba, E. G. (2013). The Constructivist Credo. Walnut Creek California, United States of America: Left Coast Press, Inc.

Matobo, T. A., Makatsa, M., \& Obioha, E. E. (2009). Continuity in the traditional initiation practice of boys and girls in contemporary Southern Africa society. Studies of Tribes and Tribals, 7(2), 105-113. https://doi.org/10.1080/0972639X.2009.11886600

Maternal \& Child Health in Lesotho. (2019). Retrieved from: https://www.jliedn.com/blog/maternal-child-health-Lesotho.

Ngomane, S., \& Mulaudzi, M. F. (2010). Indigenous beliefs and practices that influence the delayed attendance of antenatal clinics by women in the Bohlabelo district in Limpopo, South Africa. https://doi.org/10.2016/j.mid.2010.11.002

Nyathi, L.,Tugli, A.K..,Tshitangano,T.G., \& Mpofu,M (2017). Investigating the accessibility factors that influence antenatal care services utilization in mangwe district, Zimbabwe. https://doi.org/10.4102/phcfm.v9i1.1337

Oakley, L., Gray, R., Kurinczuk, J. J., Brocklehurst, P., \& Hollowell, J. (2009). A systematic review of the effectiveness of interventions to increase the early initiation of antenatal care in socially disadvantaged and vulnerable women: Final Report. Retrieved https://www.npeu.ox.ac.uk/downloads/files/infant-mortality/Infant-Mortality-ANC-Uptake-Review.pdf

Ochako, R., Fotso, J. C., IKAMARI, L., \& Khasakhala, A. (2011). Utilization of maternal health services among young women in Kenya: Insights from Kenya Demographic and health survey, 2003. BMC pregnancy \& Childbirth. https://doi.org/10.1186/1471-2393-11-1 
Onta, S., Choulagai, B., Shrestha, B., Subedi, N., Bhandari, G. P., \& Krettek, A. (2014). Perceptions of users and providers on barriers to utilizing skilled birth care in mid- and far-western Nepal: a qualitative study. Global Health Action, 7(1), 1-9. https://doi.org/10.3402/gha.v7.24580

Oyerinde, K. (2013). Can Antenatal Care Result in Significant Maternal Mortality Reduction in Developing Countries? Community Medicine \& Health Education, 3(2), 1-2. https://doi.org/10.4172/2161-0711.1000e116

Pell, C., Men, A., Were, F., Nana, A., Afrah, N. A., Chatio, S., \& Poo, R. (2013). Factors affecting antenatal care attendance: Results from qualitative studies in Ghana, Kenya and Malawi. Public Library of Science, 8(1), 1-10. https://doi.org/10.1371/journal.pone.0053747

Polit, D. F., \& Beck, C. T. (2012). Nursing Research: Generating and Assessing Evidence for Nursing Practice (9th edition). Philadelphia, United States of America: Lippincott Williams \& Wilkins.

Price Water Coopers. (2013). Health System Innovation in Lesotho. Design and Early Operations of Maseru public-private integrated partnership. Retrieved from www.pwc.com/global-health.

Resty, N. (2011). Factors affecting the utilization of antenatal care services among adolescent pregnant mothers. Case study of Naguru Teenage Health Centre, Kampala, Uganda. Retrieved from http://www.2011.isiproceedings.org/papers/950879.pdf.

Roller, M. R., \& Lavrokas, P. J. (2015). Applied qualitative research design. A total quality framework approach. Retrieved from https://psycnet.apa.org/record/2015-17413-000.

Sanda, H. U. (2014). Media awareness and utilization of antenatal care services. Retrieved from https://www.semanticscholar.org.paper/media

Shale, M. (2013). Return, reintegration and survival: The case of Basotho. Retrieved from https://www.researchgatenet/publications/288828495.

Satti, H., Motsamai S., Chetane P., Marumo, L., Barry, D.J., ... \& Mukhere, J. S. (2012). Comprehensive approach to improving maternal Health and achieving MDS 5: Report from the mountains of Lesotho. https://doi.org/10.1371/journal.pone.0042700

Semenya, D. K., \& Letsosa, R. (2013). Effects and impact of witchcraft on Sotho reformed churches and biblical view on witchcraft. Verum et Ecclesia, 34(1), 1-9. https://doi.org/10.4102/ve.v34i1.676

Shamu, S., Zarowsky, C., Shefer, T., Temmerman, M., \& Abrahams, N. (2014). Intimate partner violence after discloser of HIV test results among pregnant women in Harare, Zimbabwe. PLoS ONE, 9(10), 1-8. https://doi.org/10.1371/journal.pone.0109447

Sibiya, M. N., Ngxongo, T. S. P., \& Bhengu, T. J. (2018). Access and utilization of antenatal care services in a rural community of eThekwini district Kwazulu-Natal. International Journal of African Studies, 8(2018)1-7. https://doi.org/10.1016/j.ijans.2018.01.002

Sinyange, N., Sitali, L., Jacobs, C., Musonda, P., \& Michelo, P. (2016). Factors associated with late antenatal care booking: population based observation from 2007 Zambia demographic and health survey. https://doi.org/10.11604/pamj.2016.25.109.6873

St James Mission Hospital. The Anglican Diocese of Lesotho (2021). Retrieved from Lesotho.tacosa.org/programmes/st-james-mission-hospital html.

St James Mission Hospital. Mantsonyane Health Service area (2021). Retrieved from Lesotho.tacosa.org/programmes/st-james-mission-hospital html.

South Africa Department of Health. (2017). Improving Antenatal Care in South Africa. Retrieved from http://www.dohgov.za/docs/reports-f.html.

South Africa Department of statistics. (2015). Millennium goals country report 2015. Retrieved from http://wwwStatssa.gov.za/MDG/MDG-country\%20report-Final 30Sep 2015.pdf.

World Health Organization. (2016). Recommendations on antenatal care for a positive pregnancy experience. Retrieved

from https://apps.who.int/ins/bitstream/handle/10665/250796/97892415549912-eng.pdf?sequence=1

World Health Organization. (2015). Maternal mortality and morbidity in the united State of America. Bulletin of the World Health Organization. https://doi.org/10.2471/BlT.14.148627. 
Yanikkerem, E., Ozdemir, M., Bingol, H., Tatar, A., \& Karadeniz, G. (2009). Women's attitudes and expectations regarding gynaecological examination. NIH-PA Author Manuscript, 25(5), 500-508. https://doi.org/10.1016/j.midw.2007.08.006

Ye, Y., Yoshida, Y., Rashid, M. D., \& Sakamoto, J. (2010). Factors affecting the utilization of antenatal care services among women in Kham district, Xiengkhouang province, LAO PDR. Nagoya Journal of Medical Science, 72(1-2), 23-33.

\section{Copyrights}

Copyright for this article is retained by the author(s), with first publication rights granted to the journal.

This is an open-access article distributed under the terms and conditions of the Creative Commons Attribution license (http://creativecommons.org/licenses/by/4.0/). 